\title{
Proposing a New Theorem To Determine If an Algebraic Polynomial Is Nonnegative in an Interval
}

\author{
Ke-Pao Lin ${ }^{1}{ }^{(D)}$, Yi-Fan Wang ${ }^{2}$, Ruo-Yu Wang ${ }^{3, *}$ and Andrew Yang ${ }^{4}$ \\ 1 Department of Liberal Arts, Chang Gung University of Science and Technology, Chang Gung Memorial \\ Hospital, Tao-Yuan 33303, Taiwan; kplin@mail.cgust.edu.tw \\ 2 Institute of Information and Decision Sciences, National Taipei University of Business, \\ Taipei City 100, Taiwan; yfwang_tw@ntub.edu.tw \\ 3 School of Mathematics Sciences, Beihang University, Beijing 102206, China \\ 4 Department of Mathematics, Swansea University, Swansea SA2 8PP, UK; a.yang.852083@swansea.ac.uk \\ * Correspondence: WangRY@buaa.edu.cn
}

Citation: Lin, K.-P.; Wang, Y.-F.; Wang, R.-Y.; Yang, A. Proposing a New Theorem To Determine If an Algebraic Polynomial Is Nonnegative in an Interval. Mathematics 2021, 9, 167. https://doi.org/10.3390/ math9020167

Received: 23 November 2020 Accepted: 13 January 2021 Published: 15 January 2021

Publisher's Note: MDPI stays neutral with regard to jurisdictional clai$\mathrm{ms}$ in published maps and institutional affiliations.

Copyright: (C) 2021 by the authors. Licensee MDPI, Basel, Switzerland. This article is an open access article distributed under the terms and conditions of the Creative Commons Attribution (CC BY) license (https:// creativecommons.org/licenses/by/ $4.0 /)$.

\begin{abstract}
We face the problem to determine whether an algebraic polynomial is nonnegative in an interval the Yau Number Theoretic Conjecture and Yau Geometric Conjecture is proved. In this paper, we propose a new theorem to determine if an algebraic polynomial is nonnegative in an interval. It improves Wang-Yau Lemma for wider applications in light of Sturm's Theorem. Many polynomials can use the new theorem but cannot use Sturm's Theorem and Wang-Yau Lemma to judge whether they are nonnegative in an interval. New Theorem also performs better than Sturm's Theorem when the number of terms and degree of polynomials increase. Main Theorem can be used for polynomials whose coefficients are parameters and to any interval we use. It helps us to find the roots of complicated polynomials. The problem of constructing nonnegative trigonometric polynomials in an interval is a classical, important problem and crucial to many research areas. We can convert a given trigonometric polynomial to an algebraic polynomial. Hence, our proposed new theorem affords a new way to solve this classical, important problem.
\end{abstract}

Keywords: algebraic polynomial; trigonometric polynomials; nonnegative; Wang-Yau Lemma; Sturm's Theorem; Yau Number Theoretic Conjecture; Yau Geometric Conjecture; integral points

\section{Introduction}

Due to the application in singularity theory and number theory, the problem of computing positive lattice points in $n$-dimensional simplexes has aroused the interest of mathematicians. In recent years, great progress has been made in the calculation of the number of integral points in n-dimensional simplex with non-integral vertice (cf. [1,2]). Such an estimate could be applied to find large gaps between primes, to Waring's problem, to primality testing and factoring algorithms, to singularity theory (cf. [3]) and many others (e.g., classification theory of singularities (cf. [4]); characterization of weighted homogeneity (cf. [5-7]); Bernoulli Polynomials (cf. [8]); PDE (cf. [9,10]). For more details concerning a recent progress in this rapidly growing area of counting positive lattice points in $n$-dimensional simplexes, the interested reader is referred to [11-13]. Stephen S.-T. Yau proposes Yau Number Theoretic Conjecture and Yau Geometric Conjecture (cf. [14]) to solve the above-mentioned problems. The novelty of Yau Number Theoretic Conjecture is that we can count the integral points in a polytope whose vertices are not necessarily integer points. Proving the above-mentioned conjectures needs to prove many complicated polynomials are nonnegative in an interval. For example, proving 8 dimension Yau Number Theoretic Conjecture (cf. [15]), we need to prove many complicated 8th degree polynomials are nonnegative in an interval. The coefficients for each term of the polynomials are complicated rational function of many parameters. We use Wang-Yau Lemma (cf. [16]) and Sturm's Theorem to prove whether many complicated polynomials are nonnegative in 
an interval for 8 dimension case. It is much easier to use Wang-Yau Lemma than Sturm's Theorem, but the condition to use it is too restricted and, hence, influences the range of its application. The Main Theorem in Section 3 will cover the improvement of Wang-Yau Lemma and shows how to increase its range of application.

Yau Number Theoretic Conjecture. Let

$$
P_{n}=P_{n}\left(a_{1}, a_{2}, \ldots, a_{n}\right)=\#\left\{\left(x_{1}, \ldots, x_{n}\right) \in Z_{+}^{n}: \frac{x_{1}}{a_{1}}+\frac{x_{2}}{a_{2}}+\cdots+\frac{x_{n}}{a_{n}} \leq 1\right\},
$$

where $n \geq 3, a_{1} \geq a_{2} \geq \cdots \geq a_{n}>1$ are real numbers, $\#\{\ldots\}$ denotes the number of elements in the set. If $P_{n}>0$, then

$$
n ! P_{n} \leq \prod_{i=1}^{n}\left(a_{i}-1\right)-\left(a_{n}-1\right)^{n}+\prod_{i=0}^{n-1}\left(a_{n}-i\right)
$$

and equality holds if and only if $a_{1}=a_{2}=\cdots=a_{n}$ are integers.

Yau Geometric Conjecture. Let $f:\left(C^{n}, 0\right) \rightarrow(C, 0)$ be a weighted homogeneous polynomial with an isolated singularity at the origin. Let $\mu, p_{g}$ and $v$ be the Milnor number, geometric genus and multiplicity of singularity $V=\{z: f(z)=0\}$, respectively. Then

$$
\mu-h(v) \geq n ! p_{g}
$$

where $h(v)=(v-1)^{n}-v(v-1) \ldots(v-n+1)$. The equality holds if and only if $f$ is $a$ homogeneous polynomial after a biholomorphic change of coordinates.

For recent progress of Yau Number Theoretic Conjecture, one can see the cited references [15,17]. The Yau Geoemtric Conjecture has been proven for $3 \leq n \leq 6$ (cf. [14,18]). We believe that our Main Theorem (Theorem 1) will play an important role in the proof of these two Yau conjectures.

Example 1 illustrates some examples that the Main Theorem can judge whether the polynomial is nonnegative in an interval but Sturm's Theorem and Wang-Yau Lemma cannot do the same. It is obvious we can find infinitely many polynomials can only use the Main Theorem to judge whether they are nonnegative in an interval but cannot use Sturm's Theorem and Wang-Yau Lemma to get the same results.

Example 2 illustrates the Main Theorem is much better for polynomials of many terms and high degree. When the number of terms and degree of polynomials increase Main Theorem performs dramatically better than Sturm's Theorem. Take $P_{11}(x)$ as example. If Main Theorem needs 1 min for result then Sturm's Theorem uses about $3 \mathrm{~h}$ to do the same job.

Examples 3 and 4 are taken from Kwong's paper (cf. [19]). They show how to convert a given trigonometric polynomial to an algebraic polynomial. Then one can apply Main Theorem or Sturm's Theorem to judge whether the polynomial is nonnegative in the interval.

Example 4 considers a polynomial whose coefficients are irrational numbers (cf. [19]). It is worth noting that we cannot solve this example by Sturm's Theorem in Maple, because the coefficients of this polynomial are irrational numbers. Main Theorem can be used for polynomials with irrational coefficients like this example.

Example 5 considers a polynomial whose coefficients are parameters. It's very useful and important in the proof of Yau conjectures.

Example 6 illustrates how to apply Main Theorem to any interval we use and how it helps us to find the roots of polynomials.

We can combine Main Theorem with Newton method or bisection method to find roots of polynomials or find roots of those series which can be transformed to polynomials (for instance cosine polynomials). In practice, we can convert a given trigonometric 
polynomial to an algebraic polynomial by using Maple. Hence, to determine whether an algebraic polynomial is nonnegative in an interval is closely related to whether a trigonometric sum is nonnegative in an interval. The construction of nonnegative or positive trigonometric sums in an interval is an old problem and has been dealt with by many mathematicians (cf. $[19,20])$. The most familiar examples are the Fejér-JacksonGronwall inequality and Young's inequality. Indeed, these inequalities have remarkable applications in the approximation theory, positive quadrature methods, the theory of univalent functions, positive Jacobi polynomial sums, orthogonal polynomials on the unit circle, zero-free regions for the Riemann zeta-function, distributions for circular random variable, Gibbs phenomenon of the the Fourier series, zero-free regions for the Riemann zeta-function, just to mention a few.

\section{The Review of Previous Works}

The following two lemmas can be used to identify the nonnegative of polynomials in some restricted domains. Wang-Yau Lemma is easy to use, because when judging whether a polynomial is nonnegative, Wang-Yau Lemma only needs to sum the coefficients of the polynomial. However, the condition to use Wang-Yau Lemma is too restricted. It has happened more than once in our quest for a general criterion that the arguments we devised work well for polynomials of high degrees, but not for a finite collection of small values of dimension. Sturm's Theorem can then be used to complete the proof. In addition, the proof of a general result may require a few trigonometric inequalities that can be proved using the Sturm procedure. Sturm's Theorem is used as the last choice because it is difficult to use if not use software like Maple. In addition, when the polynomials have irrational coefficients, Maple cannot handle such case and the work will become much harder. That's why we try hard to improve Wang-Yau Lemma and get the Main theorem that can be used in much wider area. Wang-Yau Lemma is needed in the proof of the Main Theorem.

Lemma 1 (Wang-Yau Lemma). ([16] Lemma 3.1) Let $f(\beta)$ be a polynomial defined by

$$
f(\beta)=\sum_{i=0}^{n} c_{i} \beta^{i}
$$

where $\beta \in(0,1)$. If for any $k=0,1, \ldots, n$

$$
\sum_{i=0}^{k} c_{i} \geq 0
$$

then $f(\beta) \geq 0$ for $\beta \in(0,1)$.

Lemma 2 (Sturm's Theorem). Starting from a given polynomial $X=f(x)$, let the polynomials $X_{1}, X_{2}, \ldots, X_{r}$ be determined by Euclidean algorithm as follows:

$$
\begin{aligned}
X_{1} & =f^{\prime}(x) \\
X & =Q_{1} X_{1}-X_{2}, \\
X_{1} & =Q_{2} X_{2}-X_{3}, \\
\cdots & \cdots \cdots \\
X_{r-1} & =Q_{r} X_{r}
\end{aligned}
$$

where $\operatorname{deg} X_{k}>\operatorname{deg} X_{k+1}$ for $k=1, \ldots, r-1$. For every real number a which is not a root of $f(x)$ let $w(a)$ be the number of variations in sign in the number sequence

$$
X(a), X_{1}(a), \ldots, X_{r}(a)
$$


in which all zeros are omitted. If $b$ and $c$ are any numbers $(b<c)$ for which $f(x)$ does not vanish, then the number of the various roots in the interval $b \leq x \leq c$ (multiple roots to be counted only once) is equal to

$$
w(b)-w(c)
$$

Proof. See [21].

It is obvious that the computation in Lemma 2 is much more complicated than that in Lemma 1. We prefer Lemma 1 when it works.

\section{Proof of the Main Theorem}

Lemma 3. Consider a polynomial

$$
P(x)=d-x+\delta_{1} x^{2}+\delta_{2} x^{3}+\cdots+\delta_{n-1} x^{n}+\left(1-d-\delta_{1}-\delta_{2}-\cdots-\delta_{n-1}\right) x^{n+1},
$$

where $0<d<1, \delta_{i}, i=1, \cdots, n-1$ are constants satisfying

$$
d+\delta_{1}+\delta_{2}+\cdots+\delta_{l}<1, l=1, \cdots, n-1
$$

If

$$
(n+1) d+(n-1) \delta_{1}+(n-2) \delta_{2}+\cdots+\delta_{n-1}-n \geq 0,
$$

then $P(x) \geq 0$ for $0 \leq x \leq 1$.

\section{Proof.}

$$
\begin{aligned}
P(x)= & d\left(1-x^{n+1}\right)+\left(x^{n+1}-x\right)+\delta_{1}\left(x^{2}-x^{n+1}\right)+\delta_{2}\left(x^{3}-x^{n+1}\right)+\cdots+\delta_{n-1}\left(x^{n}-x^{n+1}\right) \\
= & d(1-x)\left(1+x+x^{2}+\cdots+x^{n}\right)-x\left(1-x^{n}\right)+\delta_{1} x^{2}\left(1-x^{n-1}\right)+\delta_{2} x^{3}\left(1-x^{n-2}\right) \\
& +\cdots+\delta_{n-1} x^{n}(1-x) \\
= & d(1-x)\left(1+x+x^{2}+\cdots+x^{n}\right)-x(1-x)\left(1+x+\cdots+x^{n-1}\right) \\
& +\delta_{1} x^{2}(1-x)\left(1+x+\cdots+x^{n-2}\right)+\cdots+\delta_{n-1} x^{n}(1-x) \\
= & (1-x)\left[d\left(1+x+x^{2}+\cdots+x^{n}\right)-\left(x+x^{2}+\cdots+x^{n}\right)+\delta_{1}\left(x^{2}+x^{3}+\cdots+x^{n}\right)\right. \\
& \left.+\delta_{2}\left(x^{3}+x^{4}+\cdots+x^{n}\right)+\cdots+\delta_{n-1} x^{n}\right] \\
= & (1-x)\left[d+(d-1) x+\left(d+\delta_{1}-1\right) x^{2}+\left(d+\delta_{1}+\delta_{2}-1\right) x^{3}\right. \\
& \left.+\cdots+\left(d+\delta_{1}+\delta_{2}+\cdots+\delta_{n-1}-1\right) x^{n}\right] \\
= & (1-x) g(x),
\end{aligned}
$$

where $g(x)=d+(d-1) x+\left(d+\delta_{1}-1\right) x^{2}+\left(d+\delta_{1}+\delta_{2}-1\right) x^{3}+\cdots+\left(d+\delta_{1}+\delta_{2}+\right.$ $\left.\cdots+\delta_{n-1}-1\right) x^{n}$. Note that all coefficients of $x^{k}$ with $k=1, \cdots, n$ are negative so that $g(x)$ is a decreasing function. Hence

$$
g(x) \geq 0 \Leftrightarrow g(1) \geq 0 \Leftrightarrow(n+1) d+(n-1) \delta_{1}+(n-2) \delta_{2}+\cdots+\delta_{n-1}-n \geq 0 .
$$

This completes the proof of the lemma.

Theorem 1 (Main Theorem). Consider a polynomial $f(x)=C_{0}+C_{1} x+C_{2} x^{2}+\cdots+C_{n} x^{n}$. Assume

$$
\sum_{i=0}^{k} C_{i} \geq 0, k=0,1, \cdots, m, m+l+1, \cdots, n
$$


and

$$
\sum_{i=0}^{k} C_{i}<0, k=m+1, \cdots, m+l .
$$

Denote

$$
\begin{gathered}
\sum_{i=0}^{m} C_{i}=d\left|C_{m+1}\right| \\
C_{m+2}=\delta_{1}\left|C_{m+1}\right|, C_{m+3}=\delta_{2}\left|C_{m+1}\right|, \cdots, C_{m+l}=\delta_{l-1}\left|C_{m+1}\right|
\end{gathered}
$$

If

$$
(l+1) d+(l-1) \delta_{1}+(l-2) \delta_{2}+\cdots+\delta_{l-1}-l \geq 0,
$$

then $f(x) \geq 0$ for $x \in[0,1]$.

Proof. Firstly, note that under the assumption of the theorem, we have

$$
C_{m+1}<0,
$$

moreover,

$$
d+\delta_{1}+\cdots+\delta_{k}<1, k=1, \cdots, l-1 .
$$

Now rewrite the polynomial $f(x)$ as

$$
\begin{aligned}
f(x)= & C_{0}+C_{1} x+\cdots+C_{m} x^{m}+C_{m+1} x^{m+1}+\cdots+C_{m+l} x^{m+l}+C_{m+l+1} x^{m+l+1} \\
& +\cdots+C_{n} x^{n} \\
= & C_{0}+C_{1} x+\cdots+\left(C_{m}-d\left|C_{m+1}\right|\right) x^{m}+0+\cdots+0 \\
& +\left(C_{m+l+1}-\left(1-\left(d+\delta_{1}+\cdots+\delta_{l-1}\right)\right)\left|C_{m+1}\right|\right) x^{m+l+1}+\cdots+c_{n} x^{n} \\
& +d\left|C_{m+1}\right| x^{m}+C_{m+1} x^{m+1}+\cdots+C_{m+l} x^{m+l}+\left(1-\left(d+\delta_{1}+\cdots+\delta_{l-1}\right)\right)\left|C_{m+1}\right| x^{m+l+1} \\
= & g(x)+h(x),
\end{aligned}
$$

where

$$
\begin{aligned}
h(x)= & d\left|C_{m+1}\right| x^{m}-\left|C_{m+1}\right| x^{m+1}+\delta_{1}\left|C_{m+1}\right| x^{m+2}+\cdots+\delta_{l-1}\left|C_{m+1}\right| x^{m+l} \\
& +\left(1-\left(d+\delta_{1}+\cdots+\delta_{l-1}\right)\right)\left|C_{m+1}\right| x^{m+l+1} \\
= & \left|C_{m+1}\right| x^{m}\left[d-x+\delta_{1} x^{2}+\delta_{2} x^{3}+\cdots+\delta_{l-1} x^{l}+\left(1-\left(d+\delta_{1}+\cdots+\delta_{l-1}\right)\right) x^{l+1}\right] .
\end{aligned}
$$

By Lemma $3, h(x) \geq 0$ for $x \in[0,1]$ under the assumption of the Theorem. We now turn to show that $g(x) \geq 0$ for $x \in[0,1]$.

By using the results of Wang-Yau Lemma. Rewrite $g(x)$ as

$$
\begin{aligned}
g(x)= & C_{0}+C_{1} x+\cdots+\left(C_{m}-d\left|C_{m+1}\right|\right) x^{m}+0+\cdots+0 \\
& +\left(C_{m+l+1}-\left(1-\left(d+\delta_{1}+\cdots+\delta_{l-1}\right)\right)\left|C_{m+1}\right|\right) x^{m+l+1}+C_{m+l+2} x^{m+l+2} \\
& +\cdots+C_{n} x^{n} \\
= & \bar{C}_{0}+\bar{C}_{1} x+\cdots+\bar{C}_{m} x^{m}+\bar{C}_{m+1} x^{m+1}+\cdots+\bar{C}_{m+l} x^{m+l}+\bar{C}_{m+l+1} x^{m+l+1} \\
& +\bar{C}_{m+l+2} x^{m+l+2}+\cdots+\bar{C}_{n} x^{n}
\end{aligned}
$$

where

$$
\begin{gathered}
\bar{C}_{i}=C_{i}, i=0,1, \cdots, m-1, m+l+2, \cdots, n, \\
\bar{C}_{m}=C_{m}-d\left|C_{m+1}\right|, \bar{C}_{m+1}=\& \cdots=\bar{C}_{m+l}=0, \\
\bar{C}_{m+l+1}=C_{m+l+1}-\left(1-\left(d+\delta_{1}+\cdots+\delta_{l-1}\right)\right)\left|C_{m+1}\right| .
\end{gathered}
$$


Straightforward calculation shows that

$$
\begin{aligned}
\sum_{i=0}^{k} \bar{C}_{i} & \geq 0, k=0, \cdots, m-1 \\
\sum_{i=0}^{m} \bar{C}_{i} & =\sum_{i=0}^{m-1} \bar{C}_{i}+\bar{C}_{m}=\sum_{i=0}^{m-1} C_{i}+C_{m}-d\left|C_{m+1}\right|=\sum_{i=0}^{m} C_{i}-d\left|C_{m+1}\right|=0 \geq 0, \\
\sum_{i=0}^{m+1} \bar{C}_{i} & =\cdots=\sum_{i=0}^{m+l} \bar{C}_{i}=0 \geq 0 \\
\sum_{i=0}^{m+l+1} \bar{C}_{i} & =\sum_{i=0}^{m+l} \bar{C}_{i}+\bar{C}_{m+l+1} \\
& =C_{m+l+1}-\left|C_{m+1}\right|+d\left|C_{m+1}\right|+\delta_{1}\left|C_{m+1}\right|+\cdots+\delta_{l-1}\left|C_{m+1}\right| \\
& =C_{m+l+1}+C_{m+1}+\sum_{i=0}^{m} C_{i}+C_{m+2}+C_{m+3}+\cdots+C_{m+l} \\
& =\sum_{i=0}^{m+l+1} C_{i} \\
& \geq 0 \\
\sum_{i=0}^{k} \bar{C}_{i} & =\sum_{i=0}^{k} C_{i} \geq 0, \text { for } k \geq m+l+2 .
\end{aligned}
$$

Hence $g(x) \geq 0$. Therefore $f(x)=g(x)+h(x) \geq 0$ and completes the proof of the main theorem.

The theorem holds also for a polynomial when the summations of the coefficients become negative in arbitrary sections, and the assumption in the theorem holds for the coefficients in each section.

\section{Application Examples}

Example 1. This example illustrates by three polynomials the Main Theorem can but Sturm's Theorem and Wang-Yau Lemma cannot judge whether the polynomial is nonnegative in an interval. Note that Sturm's Theorem requires that the value of the polynomial is not zero at the end of the interval. It is obvious we can find infinitely many polynomials can use the Main Theorem but cannot use Sturm's Theorem and Wang-Yau Lemma to judge whether they are nonnegative in an interval. Consider

$$
P_{1}(x)=1+x+x^{2}-\frac{7}{2} x^{3}-\frac{1}{2} x^{4}+x^{5}, x \in[0,1] .
$$

It's easy to find that $P_{1}(1)=0$. Because the Sturm's Theorem requires that the endpoint value of the polynomial cannot be zero, thus we can't use the Sturm's Theorem.

In $P_{1}(x)$,

$$
\begin{gathered}
C_{0}=1, C_{1}=1, C_{2}=1, C_{3}=-\frac{7}{2}, C_{4}=-\frac{1}{2}, C_{5}=1, \\
C_{0}=1, C_{0}+C_{1}=2, C_{0}+C_{1}+C_{2}=3, C_{0}+C_{1}+C_{2}+C_{3}=-\frac{7}{2} \\
C_{0}+C_{1}+C_{2}+C_{3}+C_{4}=-1, C_{0}+C_{1}+C_{2}+C_{3}+C_{4}+C_{5}=0
\end{gathered}
$$

Then

$$
\sum_{i=0}^{k} C_{i}<0, \text { for } k=3,4
$$


Wang-Yau Lemma requires for every $k=0,1, \ldots, n, \sum_{i=0}^{k} C_{i} \geq 0$, so we cannot use Wang-Yau Lemma to judge whether this polynomial is nonnegative.

Apply Main Theorem to $P_{1}(x)$, we get

$$
\begin{gathered}
\sum_{i=0}^{k} C_{i} \geq 0, \text { for } k=0,1,2,5, \\
\sum_{i=0}^{k} C_{i}<0, \text { for } k=3,4 .
\end{gathered}
$$

Then we can get $m=2, l=2$.

$$
\begin{gathered}
d=\frac{\sum_{i=0}^{m} C_{i}}{\left|C_{m+1}\right|}=\frac{3}{\left|-\frac{7}{2}\right|}=\frac{6}{7}, \delta_{1}=\frac{C_{m+2}}{\left|C_{m+1}\right|}=\frac{-\frac{1}{2}}{\left|-\frac{7}{2}\right|}=-\frac{1}{7} \\
(l+1) d+\delta_{1}-l=(2+1) \times \frac{6}{7}-\frac{1}{7}-2=\frac{3}{7}>0
\end{gathered}
$$

thus, according to the Main Theorem, $P_{1}(x) \geq 0$, for $x \in[0,1]$.

The following two polynomials $P_{2}(x)$ and $P_{3}(x)$ can not use Sturm's Theorem and Wang-Yau Lemma to judge whether they are nonnegative by similar reasoning.

$$
P_{2}(x)=1+x+x^{2}-\frac{15}{4} x^{3}+\frac{1}{4} x^{4}-\frac{1}{4} x^{5}-\frac{1}{4} x^{6}+x^{7}, x \in[0,1] .
$$

We check this polynomial by the Main Theorem, we find,

$$
\begin{gathered}
m=2, l=4, \\
d=\frac{4}{5}, \delta_{1}=\frac{1}{15}, \delta_{2}=-\frac{1}{15}, \delta_{3}=-\frac{1}{15}, \\
(l+1) d+(l-1) \delta_{1}+(l-2) \delta_{2}+\delta_{3}-l=0,
\end{gathered}
$$

so we can conclude that $P_{2}(x) \geq 0$, for $x \in[0,1]$.

Then we consider a polynomial of higher degree.

$$
P_{3}(x)=1+x+x^{2}-\frac{17}{4} x^{3}+x^{4}+\frac{1}{8} x^{5}-\frac{3}{4} x^{6}+\frac{5}{8} x^{7}+\frac{1}{2} x^{8}+\frac{1}{4} x^{9}-\frac{1}{2} x^{10}, x \in[0,1] .
$$

By the Main Theorem,

$$
\begin{gathered}
m=2, l=5 \\
d=\frac{12}{17}, \delta_{1}=\frac{4}{17}, \delta_{2}=-\frac{1}{34}, \delta_{3}=-\frac{3}{17}, \delta_{4}=-\frac{5}{34}, \\
(l+1) d+(l-1) \delta_{1}+(l-2) \delta_{2}+(l-3) \delta_{3}+\delta_{4}-l=\frac{1}{17}>0,
\end{gathered}
$$

so we can conclude that $P_{3}(x) \geq 0$, for $x \in[0,1]$.

Example 2. This example compares the computing efficiency of the Sturm's Theorem and the Main Theorem by using Maple 2019. The computer we use is Intel i7-8665U CPU @ $1.90 \mathrm{GHz}$ and Windows 10, the software is Maple 2019. It illustrates the Main Theorem is much better for polynomials of many terms and high degree. When the number of terms and degree of polynomials increase Main Theorem performs dramatically better than Sturm's Theorem. For $x \in[0,1]$, consider

$$
P_{4}(x)=1+x-\frac{9}{4} x^{2}-\frac{1}{5} x^{3}+\frac{1}{2} x^{4}+\frac{1}{3} x^{5}
$$




$$
\begin{aligned}
& P_{5}(x)=\frac{1}{3}+x-\frac{20}{7} x^{2}+\frac{11}{4} x^{3}-\frac{1}{3} x^{4}-\frac{1}{4} x^{5}+\frac{11}{5} x^{6}+\frac{1}{2} x^{7}+\frac{3}{4} x^{8}+\frac{1}{6} x^{9}+x^{10}, \\
& P_{6}(x)=1+x+x^{2}-\frac{17}{4} x^{3}+x^{4}+\frac{1}{8} x^{5}-\frac{3}{4} x^{6}+\frac{5}{8} x^{7}+\frac{1}{2} x^{8}+\frac{1}{4} x^{9}-\frac{1}{2} x^{10}+\frac{2}{7} x^{12}+\frac{1}{8} x^{15}+\frac{1}{3} x^{18}+\frac{1}{2} x^{20}, \\
& P_{7}(x)=1+x+x^{2}-\frac{17}{4} x^{3}+x^{4}+\frac{1}{8} x^{5}-\frac{3}{4} x^{6}+\frac{5}{8} x^{7}+\frac{1}{2} x^{8}+\frac{1}{4} x^{9}-\frac{1}{2} x^{10}+\frac{2}{7} x^{12}+x^{16}+\frac{1}{3} x^{18} \\
& +x^{20}+\frac{1}{3} x^{21}-\frac{1}{4} x^{22}+\frac{1}{5} x^{24}+\frac{1}{8} x^{25}+\frac{2}{5} x^{27}-\frac{2}{7} x^{28}+\frac{1}{9} x^{30}-\frac{1}{8} x^{32}+\frac{2}{5} x^{35}+\frac{1}{6} x^{40}, \\
& P_{8}(x)=1+x+x^{2}-\frac{17}{4} x^{3}+x^{4}+\frac{1}{8} x^{5}-\frac{3}{4} x^{6}+\frac{5}{8} x^{7}+\frac{1}{2} x^{8}+\frac{1}{4} x^{9}-\frac{1}{2} x^{10}+\frac{1}{3} x^{11}+\frac{2}{7} x^{12}-\frac{1}{5} x^{13} \\
& +\frac{3}{4} x^{14}+\frac{1}{8} x^{15}-\frac{1}{9} x^{16}+\frac{1}{2} x^{17}+\frac{1}{3} x^{18}+\frac{2}{7} x^{19}+\frac{1}{2} x^{20}+\frac{2}{7} x^{22}-\frac{1}{7} x^{24}+\frac{1}{8} x^{26}-\frac{1}{5} x^{30} \\
& +\frac{2}{5} x^{32}+\frac{1}{2} x^{34}+\frac{2}{9} x^{36}-\frac{1}{6} x^{38}+\frac{1}{7} x^{40}+\frac{1}{5} x^{50}+\frac{1}{4} x^{60}+\frac{1}{5} x^{65}-\frac{1}{8} x^{70}+\frac{1}{9} x^{75}+\frac{1}{3} x^{80} \\
& +\frac{2}{9} x^{85}+\frac{1}{2} x^{90}-\frac{1}{7} x^{95}+\frac{1}{6} x^{100} \text {, } \\
& P_{9}(x)=1+x+x^{2}-\frac{17}{4} x^{3}+x^{4}+\frac{1}{8} x^{5}-\frac{3}{4} x^{6}+\frac{5}{8} x^{7}+\frac{1}{2} x^{8}+\frac{1}{4} x^{9}-\frac{1}{2} x^{10}+\frac{1}{3} x^{11}+\frac{2}{7} x^{12}-\frac{1}{5} x^{13} \\
& +\frac{3}{4} x^{14}+\frac{1}{8} x^{15}-\frac{1}{9} x^{16}+\frac{1}{2} x^{17}+\frac{1}{3} x^{18}+\frac{2}{7} x^{19}+\frac{1}{2} x^{20}+\frac{2}{7} x^{22}-\frac{1}{7} x^{24}+\frac{1}{8} x^{26}-\frac{1}{5} x^{30} \\
& +\frac{2}{5} x^{32}+\frac{1}{2} x^{34}+\frac{2}{9} x^{36}-\frac{1}{6} x^{38}+\frac{1}{7} x^{40}+\frac{1}{5} x^{50}+\frac{1}{4} x^{60}+\frac{1}{5} x^{65}-\frac{1}{8} x^{70}+\frac{1}{9} x^{75}+\frac{1}{3} x^{80} \\
& +\frac{2}{9} x^{85}+\frac{1}{2} x^{90}-\frac{1}{7} x^{95}+\frac{1}{6} x^{100}+\frac{1}{3} x^{110}+\frac{2}{3} x^{120}-\frac{1}{8} x^{130}+\frac{1}{7} x^{140}+\frac{1}{6} x^{150}+\frac{2}{5} x^{160} \\
& -\frac{1}{9} x^{170}+x^{180}+\frac{1}{9} x^{190}+\frac{1}{2} x^{200}+\frac{1}{3} x^{210}+\frac{2}{3} x^{220}-\frac{1}{8} x^{230}+\frac{1}{7} x^{240}+\frac{1}{6} x^{250}+\frac{2}{5} x^{260} \\
& -\frac{1}{9} x^{270}+\frac{2}{5} x^{280}+\frac{1}{9} x^{290}+\frac{1}{2} x^{300} \text {, } \\
& P_{10}(x)=1+x+x^{2}-\frac{17}{4} x^{3}+x^{4}+\frac{1}{8} x^{5}-\frac{3}{4} x^{6}+\frac{5}{8} x^{7}+\frac{1}{2} x^{8}+\frac{1}{4} x^{9}-\frac{1}{2} x^{10}+\frac{1}{3} x^{11}+\frac{2}{7} x^{12}-\frac{1}{5} x^{13} \\
& +\frac{3}{4} x^{14}+\frac{1}{8} x^{15}-\frac{1}{9} x^{16}+\frac{1}{2} x^{17}+\frac{1}{3} x^{18}+\frac{2}{7} x^{19}+\frac{1}{2} x^{20}+\frac{2}{7} x^{22}-\frac{1}{7} x^{24}+\frac{1}{8} x^{26}-\frac{1}{5} x^{30} \\
& +\frac{2}{5} x^{32}+\frac{1}{2} x^{34}+\frac{2}{9} x^{36}-\frac{1}{6} x^{38}+\frac{1}{7} x^{40}+\frac{1}{5} x^{50}+\frac{1}{4} x^{60}+\frac{1}{5} x^{65}-\frac{1}{8} x^{70}+\frac{1}{9} x^{75}+\frac{1}{3} x^{80} \\
& +\frac{2}{9} x^{85}+\frac{1}{2} x^{90}-\frac{1}{7} x^{95}+\frac{1}{6} x^{100}+\frac{1}{3} x^{110}+\frac{2}{3} x^{120}-\frac{1}{8} x^{130}+\frac{1}{7} x^{140}+\frac{1}{6} x^{150}+\frac{2}{5} x^{160} \\
& -\frac{1}{9} x^{170}+x^{180}+\frac{1}{9} x^{190}+\frac{1}{2} x^{200}+\frac{1}{3} x^{210}+\frac{2}{3} x^{220}-\frac{1}{8} x^{230}+\frac{1}{7} x^{240}+\frac{1}{6} x^{250}+\frac{2}{5} x^{260} \\
& -\frac{1}{9} x^{270}+\frac{2}{5} x^{280}+\frac{1}{9} x^{290}+\frac{1}{2} x^{300}+\frac{1}{3} x^{320}+\frac{2}{3} x^{340}-\frac{1}{8} x^{360}+\frac{1}{7} x^{380}+\frac{1}{6} x^{400}+\frac{2}{5} x^{420} \\
& -\frac{1}{9} x^{440}+\frac{2}{5} x^{460}+\frac{1}{9} x^{480}+\frac{1}{2} x^{500} \\
& P_{11}(x)=1+x+x^{2}-\frac{17}{4} x^{3}+x^{4}+\frac{1}{8} x^{5}-\frac{3}{4} x^{6}+\frac{5}{8} x^{7}+\frac{1}{2} x^{8}+\frac{1}{4} x^{9}-\frac{1}{2} x^{10}+\frac{1}{3} x^{11}+\frac{2}{7} x^{12}-\frac{1}{5} x^{13} \\
& +\frac{3}{4} x^{14}+\frac{1}{8} x^{15}-\frac{1}{9} x^{16}+\frac{1}{2} x^{17}+\frac{1}{3} x^{18}+\frac{2}{7} x^{19}+\frac{1}{2} x^{20}+\frac{2}{7} x^{22}-\frac{1}{7} x^{24}+\frac{1}{8} x^{26}-\frac{1}{5} x^{30} \\
& +\frac{2}{5} x^{32}+\frac{1}{2} x^{34}+\frac{2}{9} x^{36}-\frac{1}{6} x^{38}+\frac{1}{7} x^{40}+\frac{1}{5} x^{50}+\frac{1}{4} x^{60}+\frac{1}{5} x^{65}-\frac{1}{8} x^{70}+\frac{1}{9} x^{75}+\frac{1}{3} x^{80} \\
& +\frac{2}{9} x^{85}+\frac{1}{2} x^{90}-\frac{1}{7} x^{95}+\frac{1}{6} x^{100}+\frac{1}{3} x^{110}+\frac{2}{3} x^{120}-\frac{1}{8} x^{130}+\frac{1}{7} x^{140}+\frac{1}{6} x^{150}+\frac{2}{5} x^{160} \\
& -\frac{1}{9} x^{170}+x^{180}+\frac{1}{9} x^{190}+\frac{1}{2} x^{200}+\frac{1}{3} x^{210}+\frac{2}{3} x^{220}-\frac{1}{8} x^{230}+\frac{1}{7} x^{240}+\frac{1}{6} x^{250}+\frac{2}{5} x^{260} \\
& -\frac{1}{9} x^{270}+\frac{2}{5} x^{280}+\frac{1}{9} x^{290}+\frac{1}{2} x^{300}+\frac{1}{3} x^{310}+\frac{2}{3} x^{320}-\frac{1}{8} x^{330}+\frac{1}{7} x^{340}+\frac{1}{6} x^{350}+\frac{2}{5} x^{360} \\
& -\frac{1}{9} x^{370}+\frac{2}{5} x^{380}+\frac{1}{9} x^{390}+\frac{1}{2} x^{400}+\frac{1}{3} x^{410}+\frac{2}{3} x^{420}-\frac{1}{8} x^{430}+\frac{1}{7} x^{440}+\frac{1}{6} x^{500}+\frac{2}{5} x^{600} \\
& -\frac{1}{9} x^{700}+\frac{2}{5} x^{800}+\frac{1}{9} x^{900}+\frac{1}{2} x^{1000} .
\end{aligned}
$$

In the Table $1, t_{S}$ means the cpu time used by the Sturm's Theorem using Maple, $t_{M}$ means the cpu time of the Main Theorem using Maple. According to our tests, the cpu time needed for 
calculations are roughly as follows: using Main Theorem cpu time needed grows like linearly and using Sturm's theorem cpu time needed grows like exponentialy when the terms and degree of the polynomial increase. Take $P_{11}(x)$ as example. If Main Theorem needs 1 min for result then Sturm's Theorem uses about $3 \mathrm{~h}$ to do the same job. We believe the complexity of calculations by Main Theorem and by Sturm's theorem, respectively, can be estimated. But it is not our goal or within the interests of the paper.

Table 1. Comparison of CPU running speed between Sturm's Theorem and Main Theorem.

\begin{tabular}{ccccccccc}
\hline Polynomial & $\boldsymbol{P}_{\mathbf{4}}(\boldsymbol{x})$ & $\boldsymbol{P}_{\mathbf{5}}(\boldsymbol{x})$ & $\boldsymbol{P}_{\mathbf{6}}(\boldsymbol{x})$ & $\boldsymbol{P}_{\mathbf{7}}(\boldsymbol{x})$ & $\boldsymbol{P}_{\mathbf{8}}(\boldsymbol{x})$ & $\boldsymbol{P}_{\mathbf{9}}(\boldsymbol{x})$ & $\boldsymbol{P}_{\mathbf{1 0}}(\boldsymbol{x})$ & $\boldsymbol{P}_{\mathbf{1 1}}(\boldsymbol{x})$ \\
\hline Degree $(n)$ & 5 & 10 & 20 & 40 & 100 & 300 & 500 & 1000 \\
\hline Number of terms $(N)$ & 6 & 11 & 15 & 25 & 40 & 60 & 70 & 80 \\
\hline Sturm's Theorem $\left(t_{S}\right)$ & $0.016 \mathrm{~s}$ & $0.016 \mathrm{~s}$ & $0.016 \mathrm{~s}$ & $0.098 \mathrm{~s}$ & $0.859 \mathrm{~s}$ & $7.421 \mathrm{~s}$ & $36.531 \mathrm{~s}$ & $288.953 \mathrm{~s}$ \\
\hline Main Theorem $\left(t_{M}\right)$ & $0.016 \mathrm{~s}$ & $0.016 \mathrm{~s}$ & $0.031 \mathrm{~s}$ & $0.047 \mathrm{~s}$ & $0.156 \mathrm{~s}$ & $0.578 \mathrm{~s}$ & $0.922 \mathrm{~s}$ & $1.594 \mathrm{~s}$ \\
\hline Ratio $\left(\frac{t_{s}}{t_{M}}\right)$ & 1 & 1 & 0.52 & 2.09 & 5.51 & 12.84 & 39.62 & 181.28 \\
\hline
\end{tabular}

Example 3. Examples 3 and 4 are taken from Kwong's paper (cf. [19]). They show how to convert a given trigonometric polynomial to an algebraic polynomial. Then one can try to apply Main Theorem or Sturm's Theorem to judge whether the polynomial is nonnegative in the interval.

$$
S_{1}(x)=5+4+\cos (x)+3 \cos (2 x)+4 \cos (3 x), x \in\left[0, \frac{\pi}{2}\right] .
$$

For all integers $n, \cos (n x)$ can be expanded into a polynomial of $\cos (x)$. For example, $\cos (3 x)=4 \cos ^{3}(x)-\cos (x)=4 y^{3}-y$, where $y=\cos (x)$. Applying this to $S_{1}(x)$, we obtain

$$
S_{1}(x)=P_{12}(y)=16 y^{3}+6 y^{2}-8 y+2 .
$$

As $x$ varies from 0 to $\frac{\pi}{2}, y$ varies from 1 to 0 . According to the Main Theorem,

$$
\begin{aligned}
& m=0, l=1, \delta_{0}=1, d=\frac{1}{4}, \\
& (l+1) d+\delta_{0}-l=\frac{1}{3}>0,
\end{aligned}
$$

then $P_{12}(y) \geq 0$ for $y \in[0,1]$. Thus, $S_{1}(x) \geq 0$ for $x \in\left[0, \frac{\pi}{2}\right]$.

Example 4. Example 4 considers a polynomial whose coefficients are irrational numbers (cf. [19]). It is worth noting that we cannot solve this example by Sturm's Theorem in Maple, because the coefficients of this polynomial are irrational numbers. The Main Theorem can be used in this example.

$$
S_{2}(x)=\sin (x)+\frac{1}{2} \sin (2 x)+\frac{1}{\sqrt{2}}\left(\sin (3 x)+\frac{3}{4} \sin (4 x)\right), x \in\left[0, \frac{\pi}{2}\right] .
$$

For all integers $n, \sin (n x)$ can be expanded into a polynomial of $\cos (x)$ and $\sin (x)$. For example, $\sin (3 x)=2 \sin (x) \cos ^{2}(x)-\left(2 \cos ^{2}(x)-1\right) \sin (x)=2 y^{2} \sin (x)-\left(2 y^{2}-1\right) \sin (x)$, where $y=\cos (x)$. Let $\cos (x)=y$, after expansion, we can get

$$
S_{2}(x)=\sin (x)\left[\left(1-\frac{1}{\sqrt{2}}\right)+\left(1-\frac{3}{\sqrt{2}}\right) y+\frac{4}{\sqrt{2}} y^{2}+\frac{6}{\sqrt{2}} y^{3}\right] .
$$

Because when $x \in\left[0, \frac{\pi}{2}\right], \sin (x) \geq 0$, so if we want to discuss when $x \in\left[0, \frac{\pi}{2}\right]$, whether $S_{2}(x)$ is nonnegative, it's equal to discuss

$$
P_{13}(y)=\left(1-\frac{1}{\sqrt{2}}\right)+\left(1-\frac{3}{\sqrt{2}}\right) y+\frac{4}{\sqrt{2}} y^{2}+\frac{6}{\sqrt{2}} y^{3}, y \in[0,1],
$$


so it's equivalent to do the transformation $\cos (x)=y, \sin (x)=1$.

As $x$ varies from 0 to $\frac{\pi}{2}, y$ varies from 1 to 0 . According to the Main Theorem,

$$
\begin{aligned}
& m=0, l=1, d=\frac{\sqrt{2}-1}{3-\sqrt{2}}, \delta_{0}=1, \\
& (l+1) d+\delta_{0}-l=\frac{2 \sqrt{2}-2}{3-\sqrt{2}}>0,
\end{aligned}
$$

then $P_{13}(y) \geq 0$ for $y \in[0,1]$. Thus, $S_{2}(x) \geq 0$ for $x \in\left[0, \frac{\pi}{2}\right]$.

Example 5. Example 5 considers a polynomial whose coefficients are parameters. Consider

$$
P_{14}(x)=1+x+x^{2}-a x^{3}-\frac{1}{2} x^{4}+x^{5}, x \in[0,1] .
$$

Suppose in this interval, $P_{14}(x) \geq 0$. Apply the Main Theorem to $P_{14}(x)$, we get

$$
\begin{gathered}
m=2, l=2, \\
3-a<0, \frac{5}{2}-a<0, \frac{7}{2}-a \geq 0, \\
d=\frac{3}{|a|}, \delta_{1}=-\frac{1}{2|a|}, \\
(l+1) d+\delta_{1}-l=\frac{11}{2|a|}-2 \geq 0 .
\end{gathered}
$$

In summary, $a \in\left(3, \frac{7}{2}\right]$. Thus, we can get $P_{14}(x) \geq 0$, for $x \in[0,1], a \in\left(3, \frac{7}{2}\right]$.

Example 6. Example 6 illustrates how to apply the Main Theorem to any interval we want. Example 1 shows $P_{1}(x)=1+x+x^{2}-\frac{7}{2} x^{3}-\frac{1}{2} x^{4}+x^{5} \geq 0$, for $x \in[0,1]$. We can also test this with other intervals; for example, let us consider $x \in[-2,0]$.

When $x \in[-1,0]$, we do an affine transformation. Let $y=x+1$; then, we get

$$
P_{15}(y)=3-\frac{9}{2} y-\frac{3}{2} y^{2}+\frac{17}{2} y^{3}-\frac{11}{2} y^{4}+y^{5}, y \in[0,1],
$$

then use the Main Theorem,

$$
\begin{gathered}
m=0, l=2, d=\frac{15}{17}, \delta_{1}=-\frac{1}{3}, \\
(l+1) d+\delta_{1}-l=\frac{16}{51}>0,
\end{gathered}
$$

thus, when $y \in[0,1], P_{15}(y) \geq 0$. So $P_{1}(x) \geq 0$, for $x \in[-1,0]$.

When $x \in[-2,-1]$, using Sturm's Theorem, we get that there exists a root in this interval. Hence, in this interval, the polynomial has both positive and negative values (we assume this root is not an even multiple root). Therefore, we continue to cut this interval. Firstly, we cut this interval into five parts. After calculation, when $x$ belongs to $[-1.6,-1.4] \cup[-1.4,-1.2] \cup[-1.2,-1]$, we separately do the linear transformation $y=5\left(x+\frac{8}{5}\right), y=5\left(x+\frac{7}{5}\right), y=5\left(x+\frac{6}{5}\right)$, then apply the Main Theorem; we get $m=5, l=0$; thus, for $x \in[-1.6,-1], P_{1}(x) \geq 0$.

When $x \in[-2,-1.8]$, we do a linear transformation $y=5(x+2)$; we get

$$
P_{16}(y)=-9+\frac{51}{5} y-\frac{14}{5} y^{2}+\frac{81}{250} y^{3}-\frac{21}{1250} y^{4}+\frac{1}{3125} y^{5}, y \in[0,1],
$$


This interval is more likely to take negative values. Hence, consider that

$$
P_{17}(y)=-P_{16}(y)=9-\frac{51}{5} y+\frac{14}{5} y^{2}-\frac{81}{250} y^{3}+\frac{21}{1250} y^{4}-\frac{1}{3125} y^{5}, y \in[0,1]
$$

then applying the Main Theorem to $P_{17}(y)$, we get

$$
\begin{gathered}
m=0, l=1, d=\frac{15}{17}, \delta_{0}=1, \\
(l+1) d+\delta_{0}-l=\frac{30}{17}>0,
\end{gathered}
$$

so $P_{17}(y) \geq 0$, where $y \in[0,1]$,thus, $P_{16}(y) \leq 0$, for $y \in[0,1]$. Therefore, $P_{1}(x) \leq 0$, for $x \in[-2,-1.8]$.

When $x \in[-1.8,-1.6]$, we first use Sturm's Theorem where there exists a root in this interval. Then, we further divide the interval into $[-1.8,-1.7]$ and $[-1.7,-1.6]$.

When $x \in[-1.7,-1.6]$, we do a linear transformation $y=10\left(x+\frac{17}{10}\right)$; using Main Theorem, we get $m=5, l=0$. Thus, $P_{1}(x) \geq 0$, for $x \in[-1.7,-1.6]$.

Now only $[-1.8,-1.7]$ need to be tested. When the interval $[-1.8,-1.7]$ is cut into five parts, and then Main Theorem is used, we get the following: when $x$ belongs to $[-1.8,-1.78] \cup$ $[-1.78,-1.76], P_{1}(x) \leq 0$, when $x$ belongs to $[-1.74,-1.72] \cup[-1.72,-1.7], P_{1}(x) \geq 0$. We can find the root to any desired accuracy by using the bisection method. When the accuracy is $10^{-9}$, the root of $P_{1}(x)$ is $x=-1.748592480$, for $x \in[-2,0]$.

It is much easier to use Main Theorem to find the roots of polynomials.

\section{Conclusions}

We propose a theorem to determine whether an algebraic polynomial is nonnegative in an interval. This theorem improves Wang-Yau Lemma for Wider Applications in light of Sturm's Theorem. Infinitely many polynomials can be judged by using the new theorem but cannot be judged by using Sturm's Theorem and Wang-Yau Lemma to see whether they are nonnegative in an interval. Main Theorem performs much better than Sturm's Theorem for polynomials of many terms and high degree. Main Theorem can be used for a polynomial whose coefficients are parameters and to any interval we want. It helps us to find the roots of polynomials. To determine whether an algebraic polynomial is nonnegative in an interval is closely related to determine whether a trigonometric sum is nonnegative in an interval. Main Theorem provides a new way to solve this classical, important problem. It perfoms much better than Wang-Yau Lemma. Main Theorem is a sufficient condition. Many cases can be proved nonnegative by Sturm's Theorem but fail in our test using Main Theorem. However, Main Theorem is much easier to use than Sturm's Theorem. Therefore, we suggest making further improvements to Main Theorem in this way.

Author Contributions: formula derivation, K.-P.L. and A.Y.; software, Y.-F.W. and R.-Y.W.; example writing, R.-Y.W.; writing—original draft preparation, K.-P.L. and R.-Y.W.; writing—final draft, K.-P.L. and R.-Y.W. All authors have read and agreed to the published version of the manuscript.

Funding: This research was funded by the Ministry of Science and Technology and Chang Gung Memorial Hospital (grant no. MOST 108-2115-M-255-001, NMRPF3J0041).

Conflicts of Interest: The authors declare no conflict of interest.

\section{References}

1. Lin, K.-P.; Luo, X.; Yau, S.S.-T.; Zuo, H.Q. On number theoretic conjecture of positive integral points in 5-dimensional tetrahedron and a sharp estimation of Dickman-De Bruijn function. J. Eur. Math. Soc. 2014, 16, 90-102.

2. Lin, K.-P.; Yau, S.S.-T. Counting the number of integral points in general $n$-dimensional tetrahedra and Bernoulli polynomials. Can. Math. Bull. 2003, 46, 229-241. [CrossRef]

3. Yau, S.S.-T.; Zuo, H.Q. A sharp lower bound for the geometric genus and Zariski multiplicity question. Math. Z. 2018, 289, 1299-1310. [CrossRef] 
4. Zuo, H.Q. On strong vanishing property and plurigenera of isolated singularities. J. Algebra 2019, 529, 124-144. [CrossRef]

5. Yau, S.S.-T.; Zuo, H.Q. Lower estimate of Milnor number and characterization of isolated homogeneous hypersurface singularities. Pac. J. Math. 2012, 260, 245-255. [CrossRef]

6. Yau, S.S.-T.; Zuo, H.Q. Complete characterization of isolated homogeneous hypersurface singularities. Pac. J. Math. 2015, 273, 213-224.

7. Yau, S.S.-T.; Zuo, H.Q. Characterization of isolated complete intersection singularities with $C^{*}$-action of dimension $\geq 2$ by means of geometric genus and irregularity. Comm. Anal. Geom. 2013, 21, 509-526. [CrossRef]

8. Kang, J.Y.; Ryoo, C.S. Various Structures of the Roots and Explicit Properties of q-cosine Bernoulli Polynomials and q-sine Bernoulli Polynomials. Mathematics 2020, 8, 463. [CrossRef]

9. Ragusa, M.A.; Tachikawa, A. Regularity for minimizers for functionals of double phase with variable exponents. Adv. Nonlinear Anal. 2020, 9, 710-728.

10. Ragusa, M.A.; Scapellato, A. Mixed Morrey spaces and their applications to partial differential equations. Nonlinear Anal. Theory Methods Appl. 2017, 151, 51-65. [CrossRef]

11. Luo, X.; Yau, S.S.-T.; Zuo, H.Q. A sharp estimate of Dickman-de Bruijin function and a sharp polynomial estimate of positive integral points in 4-dimensional tetrahedron. Math. Nachr. 2015, 288, 61-75. [CrossRef]

12. Yau, S.S.-T.; Yuan, B.; Zuo, H.Q. Biggest sharp polynomial estimate of integral points in right-angled simplices. J. Number Theory 2016, 160, 254-286. [CrossRef]

13. Zhao, L.; Yau, S.S.-T.; Zuo, H.Q. Biggest sharp polynomial estimate of integral points in right-angled simplices, Topology of algebraic varietiesand singularities. Contemp. Math. AMS 2011, 538, 433-467.

14. Lin, K.-P.; Raghuvanshi, S.; Yau, S.S.-T.; Zuo, H.Q. On Yau geometric conjecture for weighted homogeneous singularities. Asian J. Math. 2018, 22, 599-646. [CrossRef]

15. Lin, K.-P.; Yang, A.; Yau, S.S.-T.; Zuo, H.Q. On the Sharp Polynomial Upper Estimate Conjecture in Eight-Dimensional Simplex. Pure Appl. Math. Q. 2018, 12, 353-398. [CrossRef]

16. Wang, X.-J.; Yau, S.S.-T. On the GLY conjecture of upper estimate of positive integral points in real right-angled simplices. J. Number Theory 2007, 122, 184-210. [CrossRef]

17. Liang, A.; Yau, S.S.-T.; Zuo, H.Q. A sharp upper estimate of positive integral points in 6-dimensional tetrahedra and smooth numbers. Sci. China Math. 2016, 59, 425-444. [CrossRef]

18. Chen, I.; Lin, K.-P.; Yau, S.S.-T.; Zuo, H.Q. Coordinate-free characterization of homogeneous polynomials with isolated singularities. Commun. Anal. Geom. 2011, 19, 661-704. [CrossRef]

19. Kwong, M.K. Nonnegative Trigonometric Polynomials, Sturms Theorem, and Symbolic Computation. arXiv 2014, arXiv:1402.6778V1.

20. Askey, I.R. Orthogonal Polynomials and Special Functions; SIAM: Philadelphia, PA, USA, 1975; Volume 21.

21. Van der Waerden, B.L. Modern Algebra; Frederick Ungar Publishing Co.: New York, NY, USA, 1950; Volume I. 